\title{
Macrophage migration inhibitory factor promotes cardiac fibroblast proliferation through the Src kinase signaling pathway
}

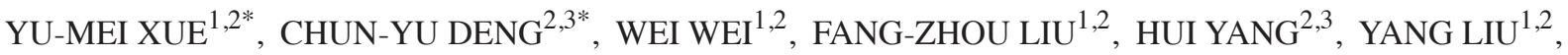 \\ XIN LI ${ }^{1,2}$, ZHAOYU WANG $^{1,2}$, SU-JUAN KUANG ${ }^{2,3}$, SHU-LIN WU $^{1-3}$ and FANG RAO ${ }^{1-3}$ \\ ${ }^{1}$ Department of Cardiology, Guangdong Cardiovascular Institute; ${ }^{2}$ Guangdong Key Laboratory of \\ Clinical Pharmacology and Medicine, Guangdong Academy of Medical Sciences; ${ }^{3}$ Research Center of \\ Medical Sciences, Guangdong General Hospital, Guangzhou, Guangdong 510080, P.R. China
}

Received July 3, 2017; Accepted October 30, 2017

DOI: $10.3892 / \mathrm{mmr} .2017 .8261$

\begin{abstract}
Atrial fibrosis is the fundamental characteristic of the structural pathology associated with atrial fibrillation (AF). Inflammation can contribute to atrial fibrosis, engendering AF. The present study aimed to investigate the role of macrophage migration inhibitory factor (MIF), a pleiotropic cytokine, in the regulation of proliferation and function of cardiac fibroblasts (CFs). Biochemical assays were performed to examine the expression of extracellular matrix (ECM) in human atrial tissues, and the proliferation and regulation of ECM induced by MIF in CFs. The expression of ECM, including collage type $3, \alpha 1$ (Col-3A1), matrix metalloproteinase (MMP)-2/-9 and transforming growth factor (TGF) $\beta$ was higher in patients with permanent AF, compared with patients in sinus rhythm (SR), and the expression levels of MIF were also increased in AF. Treatment of CFs with mouse recombinant MIF (rMIF; $40 \mathrm{nM}$ ) for $48 \mathrm{~h}$ was found to promote the proliferation of CFs. The MIF-induced CF proliferation was completely inhibited by tyrosine kinase inhibitor-PP1. rMIF treatment also stimulated the activation of Src kinase in CFs. In addition, MIF treatment upregulated the expression levels of fibrosis-related proteins, Col-1, Col-3, MMP-2/-9 and TGF- $\beta$, in the CFs. These results suggested that MIF was involved in the structural remodeling that accompanies AF, possibly by promoting the proliferation of CFs and increasing the expression of ECM. These data implicate inflammation as a potential driver of $\mathrm{CF}$.
\end{abstract}

Correspondence to: Professor Shu-Lin Wu or Dr Fang Rao, Department of Cardiology, Guangdong Cardiovascular Institute, Guangdong Academy of Medical Sciences, 96 Dongchuan Road, Guangzhou, Guangdong 510080, P.R. China

E-mail: wushulincn@126.com

E-mail: raofang@medmail.com.cn

${ }^{*}$ Contributed equally

Key words: atrial fibrosis, cardiac fibroblasts, macrophage migration inhibitory factor, proliferation, Src kinase

\section{Introduction}

Increasing data have revealed that atrial fibrosis is the fundamental characteristic of the structural pathology associated with atrial fibrillation (AF) (1-3). Fibrosis is a consequence of an imbalance between the degradation and deposition of cardiac extracellular matrix (ECM). Fibroblast proliferation and differentiation into collagen-secreting myofibroblasts contribute to atrial fibrosis via excess ECM deposition, which promotes re-entry and enhances automaticity $(4,5)$. Although it is clear that fibrosis is associated with $\mathrm{AF}$, the precise role of fibrosis in the pathogenesis of $\mathrm{AF}$ remains to be fully elucidated.

Following decades of investigations, increasing evidence has revealed that inflammation is important the pathogenesis of AF. Markers of pro-inflammatory cytokines, including C-reactive protein, tumor necrosis factor- $\alpha$ (TNF- $\alpha$ ), interleukin-6 (IL-6) and macrophage migration inhibitory factor (MIF), are increased in patients with AF (6-9). This increases the possibility that inflammation may contribute to atrial fibrosis and atrial structural remodeling, which contribute to AF. Previous studies have demonstrated that inflammation is associated with atrial fibrosis. The number of immune cells resident in tissues and higher levels of serum inflammatory markers are associated with extensive left atrium fibrosis and enlargement in patients with AF (10-12).

In the authors' previous study, it was demonstrated that MIF, an important pro-inflammatory cytokine, is involved in the electrical remodeling of atrium myocytes through regulating the $\mathrm{L}$ - and T-type calcium channel current $(8,13)$. However, the role of MIF in atrial fibrosis remains to be fully elucidated. The present study aimed to investigate whether MIF affects the proliferation and secretory functions of cardiac fibroblasts (CFs).

\section{Materials and methods}

Patients. The present study was performed in accordance with the principles outlined in the Declaration of Helsinki and was approved by the research Ethics Committee of Guangdong General Hospital, Guangdong Academy of Medical Sciences (no. GDREC2013047H; Guangzhou, China). All patients provided written informed consent (version no. 20130307). 
Patients with pneumonia or other infectious diseases were excluded from the study. Left atrial appendages were obtained from patients undergoing open-heart surgery (from March 2013 to March 2016) in Guangdong General Hospital in Guangzhou (Guangdong, China). The specimens were collected prior to initiation of cardiopulmonary bypass, immediately snap-frozen in liquid nitrogen, and stored at $-80^{\circ} \mathrm{C}$ until the day of the experiments. Tissues from 11 patients with chronic AF (from 32-67 years) and a control group of 10 patients (from 30-61 years) with sinus rhythm (SR) were also used in the present study. Patient data are presented in Table I.

Culture of primary CFs. The experiments using CFs were approved by the research Ethics Committee of Guangdong General Hospital, Guangdong Academy of Medical Sciences (no. GDREC2013047A). The CFs were isolated from 1-3-day-old Wistar rats ( $\mathrm{n}=24,14: 10$ male:female) which were provided by Sun Yat-sen University and fed at $20-25^{\circ} \mathrm{C}(12-\mathrm{h}$ light/dark cycle), as previously described (14). Briefly, the heart tissues were finely minced and mechanically digested with $0.25 \%$ Trypsin + EDTA (Gibco; Thermo Fisher Scientific, Inc., Waltham, MA, USA) using a rotor in a flask. The cell suspensions were plated in a cell culture flask for 90 min to separate the fibroblasts and cardiomyocytes. The majority of CFs adhered to the flask. The cells were cultured in Dulbecco's modified Eagle's medium (Gibco; Thermo Fisher Scientific, Inc.) containing $4.5 \mathrm{~g} / 1 \mathrm{D}$-glucose, $10 \%$ fetal bovine serum (Gibco; Thermo Fisher Scientific, Inc.), penicillin (100 U/ml) and streptomycin $(100 \mu \mathrm{g} / \mathrm{ml})$ in a humidified atmosphere (95\% humidity, $37^{\circ} \mathrm{C}$ ) composed of $95 \% \mathrm{O}_{2}, 5 \% \mathrm{CO}_{2}$. The CFs were used at passages 3-6.

Cell treatments. The cells were treated according to three experimental designs. In the first, the cells (50-60\% confluence) were treated with different doses of mouse recombinant MIF at $37^{\circ} \mathrm{C}$ (rMIF; 20 and $40 \mathrm{nM}$; R\&D Systems Inc., Minneapolis, MN, USA) for 24 or $48 \mathrm{~h}$. In the second, the cells were pretreated with PP1 (5 $\mu \mathrm{M}$; Sigma-Aldrich; Merck KGaA, Darmstadt, Germany) for $1 \mathrm{~h}$. The MIF treatment groups were pretreated with an equal quantity of dimethyl sulfoxide (DMSO) and were stimulated with $40 \mathrm{nM}$ MIF for $48 \mathrm{~h}$. In the third, the cells were treated with $40 \mathrm{nM}$ MIF for 15, 30, 45 and $60 \mathrm{~min}$, or with 20 and $40 \mathrm{nM}$ MIF for $24 \mathrm{~h}$.

Cell proliferation assay. The CFs were plated into a 96-well plate at a density of 4,500/well and proliferation was measured using the cell proliferation reagent (WST-1) kit (cat. no. 11644807001; Roche Diagnostics GmbH, Mannheim, Germany) according to the manufacturer's instructions. The signal in optical density was read at $450 \mathrm{~nm}$ on a plate reader (Mutiskan GO; Thermo Fisher Scientific, Inc.). The experiment was repeated three times.

Western blot analysis. The cells were lysed in $0.05 \mathrm{M}$ Tris- $\mathrm{HCl}$ buffer ( $\mathrm{pH} 8.0$ ), containing $0.15 \mathrm{M}$ sodium chloride, $0.02 \%$ sodium azide, $0.1 \%$ sodium dodecyl sulfate (SDS), 1\% Nonidet P-40 (NP-40), and a protease inhibitor cocktail set (Calbiochem; EMD Millipore, Billerica, MA, USA). The cell lysates were centrifuged at $12,000 \mathrm{x} \mathrm{g}$ for $15 \mathrm{~min}$ at $4^{\circ} \mathrm{C}$ and protein concentrations were determined by
Table I. Baseline characteristics of patients.

\begin{tabular}{lcc}
\hline Characteristic & SR & AF \\
\hline Patients (n) & 10 & 11 \\
Men (n) & 4 & 4 \\
Age (years) & $42.8 \pm 11.1$ & $46.9 \pm 14.4$ \\
LAD (mm) & $48.2 \pm 8.4$ & $50.1 \pm 4.4$ \\
EF (\%) & $65.0 \pm 7.3$ & $62.1 \pm 6.3$ \\
AVR (n) & 5 & 5 \\
MVR (n) & 5 & 6 \\
$\beta$-blocker (n) & 1 & 2 \\
Digitalis (n) & 1 & 6 \\
\hline
\end{tabular}

Left atrial appendages were obtained during open-heart surgery from patients with rheumatic valvular disease with chronic $\mathrm{AF}$ ( $\geq 6$ months) or SR. Age, sex distribution, type of valve disease and left ventricular function were matched. AF, atrial fibrillation; SR, sinus rhythm; $\mathrm{LAD}$, left atrial diameter; $\mathrm{EF}$, ejection fraction; AVR, aortic valve replacement; MVR, mitral valve replacement. Age, LAD and EF are presented as the mean \pm standard deviation.

bicinchoninic acid Protein Assay kit (Pierce; Thermo Fisher Scientific, Inc.). The samples were diluted with $4 \mathrm{X}$ loading buffer (Bio-Rad Laboratories, Inc., Hercules, CA, USA) and heated at $95^{\circ} \mathrm{C}$ for $5 \mathrm{~min}$. The proteins $(30 \mu \mathrm{g})$ were fractionated on $8 \%$ SDS-polyacrylamide gels for Src, phosphorylated (p-)Src, collagen type 1, $\alpha 1$ (Col-1A1), collagen type 3, $\alpha 1$ (Col-3A1), matrix metalloproteinase (MMP)-2/-9 and transforming growth factor (TGF)- $\beta$ or $10 \%$ SDS polyacrylamide gels for MIF. The proteins were then transferred onto nitrocellulose membranes (GE Healthcare Life Sciences, Chalfont, UK) according to standard protocols. The membranes were blocked with dried skimmed milk powder in TBS-Tween-20 (TBST) for $1 \mathrm{~h}$ at room temperature, prior to overnight incubation at $4{ }^{\circ} \mathrm{C}$ with primary rabbit polyclonal antibodies against Src (1:1,000, cat. no. ab47405; Abcam, Cambridge, UK), p-Src $\operatorname{Tyr}^{416}$ (1:1,000, cat. no. 2101; Cell Signaling Technology, Inc., Danvers, MA, USA), Col-1A1 (1:1,000, cat. no. ab34710), Col-3A1 (1:1,000, cat. no. ab7778) (both from Abcam), MMP-9 (1:1,000, cat. no. AB19016; EMD Millipore, Billerica, MA, USA) and TGF- $\beta$ (1:1,000, cat. no. 3711; Cell Signaling Technology, Inc.) and MMP-2 (1:2,000, cat. no. AB19015; EMD Millipore;). The signals were normalized to the protein levels of GAPDH $(1: 1,000$, cat. no. 2118) or $\beta$-actin $(1: 3,000$, cat. no. 3700) (both from Cell Signaling Technology, Inc.). Following washing in TBST, the membranes were incubated for $1 \mathrm{~h}$ with horseradish peroxidase-conjugated anti-rabbit or anti-mouse $\mathrm{IgG}$ (1:5,000-1:10,000; Abcam) in blocking solution. The protein bands were visualized using electrochemiluminescence reagents (Pierce; Thermo Fisher Scientific, Inc.), and images were evaluated densitometrically using ImageJ $1.39 \mathrm{U}$ software (National Institutes of Health, Bethesda, MD, USA).

Drugs. The rMIF was dissolved in sterile PBS containing $0.1 \%$ bovine serum albumin (Sigma-Aldrich; Merck KGaA). PP1 was purchased from Sigma-Aldrich; Merck KGaA. The 

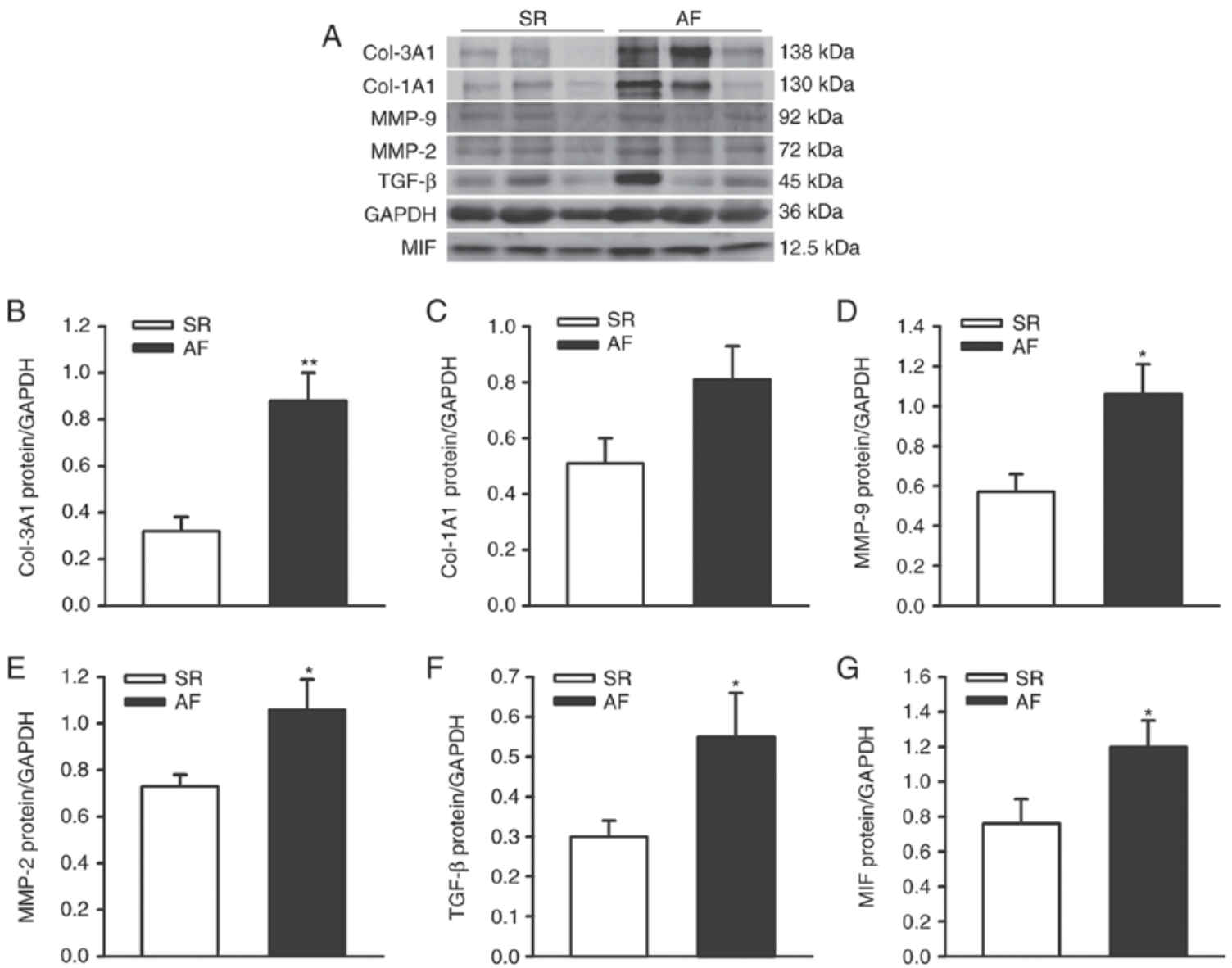

Figure 1. Expression of ECM and MIF in left atrial appendages from patients with chronic AF and SR. (A) Representative immunoblots and protein levels of ECM (Col-1A1, Col-3A1, MMP-2/-9 and TGF- $\beta$ ) and MIF in left atrial appendages from patients with AF and SR. GAPDH was used as an internal control. Densitometric analysis of protein levels of (B) Col-3A1, (C) Col-1A1, (D) MMP-9, (E) MMP-2, (F) TGF- $\beta$ and (G) MIF in patients with AF and SR controls ( $\mathrm{n}=10$ in SR group, $\mathrm{n}=11$ in AF group). ${ }^{* *} \mathrm{P}<0.01$ and ${ }^{*} \mathrm{P}<0.05$ vs. SR. AF, atrial fibrillation; SR, sinus rhythm; ECM, extracellular matrix; Col-1A1, collagen type $1, \alpha 1$; Col-3A1, collagen type $3, \alpha 1$; MMP-2/-9, matrix metalloproteinase-2/-9; TGF- $\beta$, transforming growth factor- $\beta$; MIF, migration inhibitory factor.

kinase inhibitor was dissolved in DMSO (Sigma-Aldrich; Merck KGaA). The concentration of DMSO in the working solutions did not exceed $1.0 \%$.

Statistical analysis. All data are expressed as the mean \pm standard error of the mean. Student's t-test was used as evaluate pairwise statistical significances of differences between two group means, and one-way analysis of variance was used for multiple groups with SPSS 21 statistical software (IBM Corp, Armonk, NY, USA). P $<0.05$ was considered to indicate a statistically significant difference.

\section{Results}

Expression of Col-1A1, Col-3A1, MMP-2/-9, TGF- $\beta$ and MIF in left atrial homogenates of patients with $S R$ and $A F$. The protein levels of Col-1A1, Col-3A1, MMP-2/-9, TGF- $\beta$ and MIF were detected in left atrial homogenates from patients with AF and SR controls (for patient characteristics; Table I). The protein expression levels of Col-1A1, Col-3A1, MMP-2/-9, TGF- $\beta$ and MIF were normalized to those of GAPDH in each sample. With the exception of Col-1A1, the protein levels of Col-3A1, MMP-2/9, TGF- $\beta 1$ and MIF were significantly higher in the left atrial tissues from patients with AF $(n=11)$, compared with the SR $(n=10)$ group $($ Col-1A1, 0.81 \pm 0.12 , vs. 0.51 $\pm 0.09, \mathrm{P}=0.062$; Col-3A1, 0.88 \pm 0.12 , vs. 0.32 \pm 0.06 , $\mathrm{P}=0.001$; MMP-2, 1.06 \pm 0.09 , vs . 0.73 $\pm 0.05, \mathrm{P}=0.036$; MMP-9, $1.06 \pm 0.15$, vs. $0.57 \pm 0.09, \mathrm{P}=0.013$; TGF- $\beta, 0.55 \pm 0.11$, vs. $0.30 \pm 0.04, \mathrm{P}=0.049$; MIF, $1.20 \pm 0.15$, vs. $0.76 \pm 0.14$, $\mathrm{P}=0.044$ ), as shown in Fig. $1 \mathrm{~A}-\mathrm{G}$. These results indicated that, as an important proinflammatory cytokines, MIF may be involved in the regulation of the atrial fibrosis, which contributes to the onset of AF.

MIF promotes the proliferation of CFs. The proliferation of CFs is important in myocardial fibrosis. The present study investigated the proliferation of CFs using CCK-8 assays, and it was found that 24-h MIF treatment had no effect on the proliferation of $\mathrm{CFs}$, whereas cells exhibited rapid growth following stimulation for $48 \mathrm{~h}$ with $40 \mathrm{nM}$ MIF (Fig. 2A).

Src kinase inhibitor alleviates the promoting effect of MIF on $C F$ proliferation. Src, a member of the protein tyrosine kinase (PTK) family, has been suggested to be involved in cell proliferation. The present study examined whether Src kinases were involved in MIF-induced CF proliferation using a specific Src antagonist (PP1). The CFs were pretreated with PP1 $(5 \mu \mathrm{M})$ for $1 \mathrm{~h}$ prior to rMIF stimulation. PP1 completely 

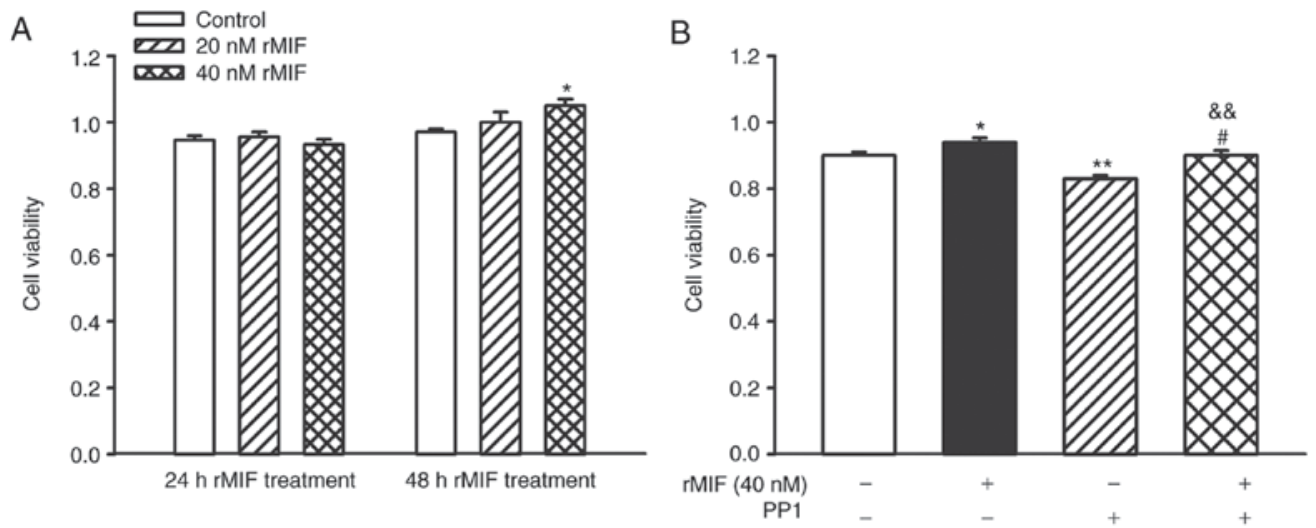

Figure 2. Effect of PP1 on the proliferation of CFs induced by rMIF. (A) Proliferation of CFs 24 or $48 \mathrm{~h}$ following rMIF treatment ( $20 \mathrm{or} 40 \mathrm{nM}$ ). The Cell Counting Kit-8 assay results demonstrated that rMIF regulated cell proliferation. Cell viability was markedly increased in the $48 \mathrm{~h} \mathrm{rMIF}(40 \mathrm{nM})$ treatment group ( $\mathrm{P}=0.017$ ). (B) Results indicated that the proliferation of CFs induced by rMIF was depressed by PP1, the protein tyrosine kinase inhibitor. Data are presented as the mean \pm standard deviation of three independent experiments. ${ }^{* *} \mathrm{P}<0.01$ and ${ }^{~} \mathrm{P}<0.05$ vs. control cells; ${ }^{\# P} \mathrm{P}<0.05$ vs. rMIF-treated cells; \&\& $\mathrm{P}<0.01$ vs. PP1-treated cells. CFs, cardiac fibroblasts; rMIF, recombinant migration inhibitory factor.

A
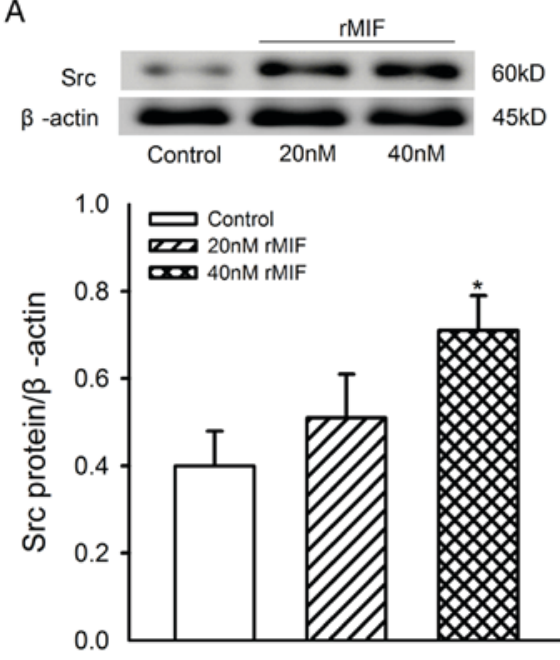
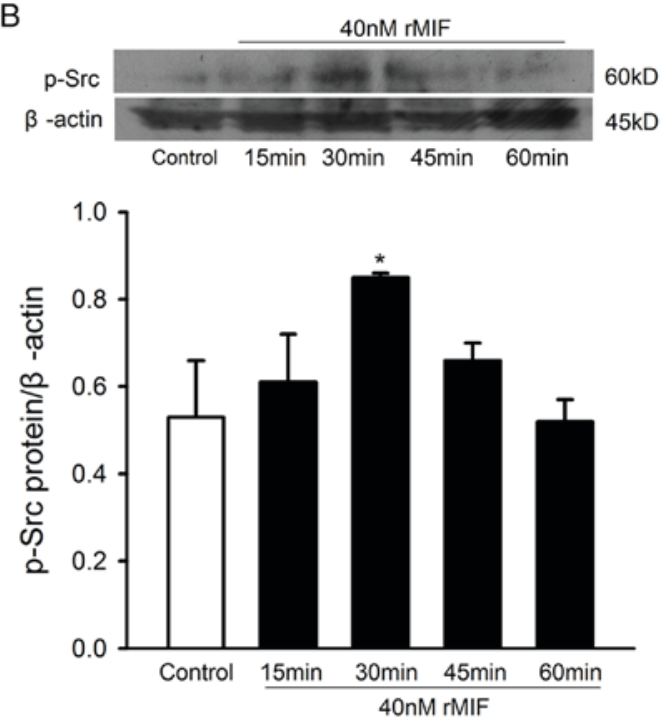

Figure 3. Expression and activation of Src in CFs following rMIF treatment. (A) Protein levels of Src in CFs following $24 \mathrm{~h}$ of rMIF treatment (20 and $40 \mathrm{nM}$ ). Representative western blot analysis (above) of Src kinase protein in treated cells; $\beta$-actin was used as the internal control. Densitometric analysis (below) of Src protein in treated cells and controls. (B) Activation of Src following treatment of cells with $40 \mathrm{nM}$ rMIF for 0, 15, 30, 45 and 60 min. Representative western blot analysis (above) of $\mathrm{p}$-Src protein in treated cells and controls; $\beta$-actin was used as the internal control. Densitometric analysis (below) of p-Src protein in treated cells and controls. " $\mathrm{P}<0.05$ vs. control. Each blot represents one of three or four experiments. CFs, cardiac fibroblasts; rMIF, recombinant migration inhibitory factor; $\mathrm{p}$, phosphorylated.

inhibited the MIF-induced CF proliferation (Fig. 2B). These results suggested that MIF induced cell proliferation through Src kinase-dependent mechanisms in CFs.

Effect of rMIF on the expression of Src in CFs. The results reported above suggested that the Src kinase signaling pathway may be responsible for the proliferation of CFs induced by rMIF. To confirm the specificity of the signaling pathways involved in the proliferation of CFs induced by rMIF, western blot analysis was performed to analyze the levels of Src and p-Src in CFs following treatment with MIF. The CFs were treated with 20 or $40 \mathrm{nM}$ of rMIF for $24 \mathrm{~h}$, or $40 \mathrm{nM}$ rMIF for $0,15,30,45$ and $60 \mathrm{~min}$, and the levels of Src and p-Src were measured. The higher concentration of $\operatorname{rMIF}(40 \mathrm{nM})$ was found to produce a marked increase in the protein levels of Src, compared with those in the unstimulated cells and the $20 \mathrm{nM}$ rMIF-treated group of cells, and Src was activated by $40 \mathrm{nM} \mathrm{rMIF}$ at $30 \mathrm{~min}$ (Fig. 3A and B).

MIF increases the levels of Col-1A1, Col-3A1, MMP-2/-9 and $T G F-\beta$ in $C F s$. To investigate the role of MIF on the secretion function of $\mathrm{CFs}$, the present study also examined the effects of MIF on collagen deposition, and the expression of MMP and profibrotic TGF- $\beta$ in CFs. The MIF dose-response was assessed using concentrations of 20 and $40 \mathrm{nM}$ for $24 \mathrm{~h}$, and it was found that the expression levels of Col-1A1, Col-3A1, MMP-2/-9 and TGF- $\beta$ increased significantly in the $40 \mathrm{nM}$ rMIF treatment group, but not in $20 \mathrm{nM} \mathrm{rMIF}$ treatment group, with the exception of MMP-9 which did increase significantly at $20 \mathrm{nM}$ (Fig. 4A-F). 
A

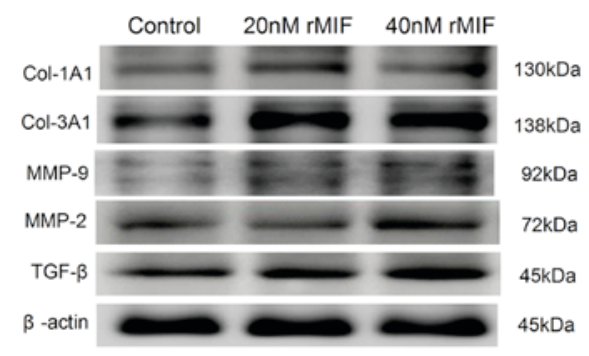

C

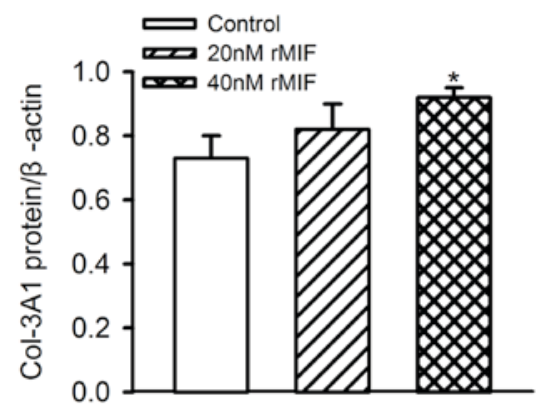

$\mathrm{E}$

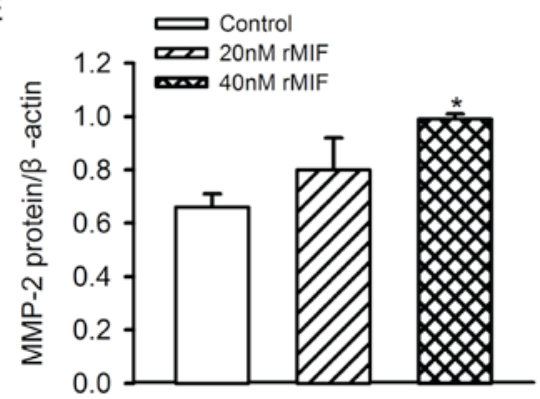

B $\rightleftharpoons$ Control

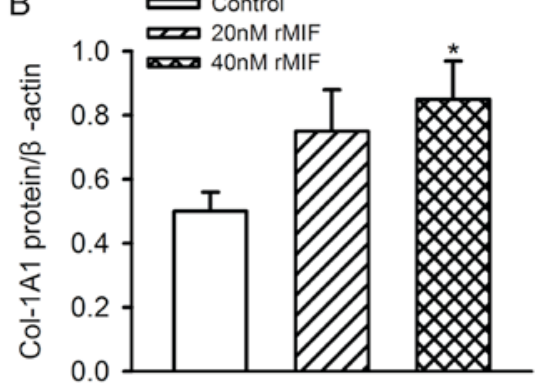

D

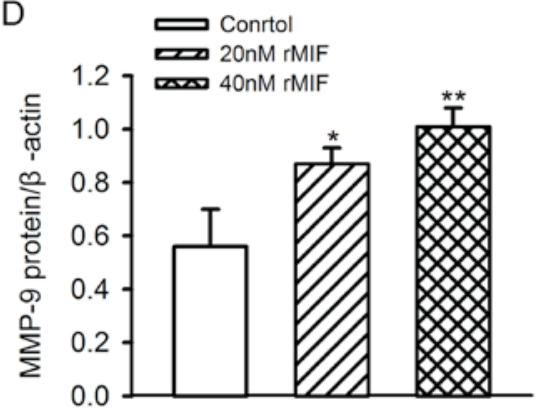

$\mathrm{F}$

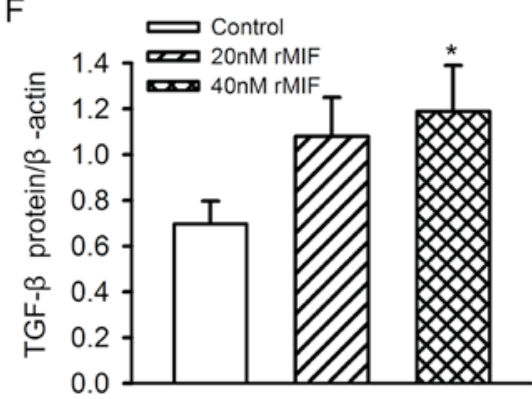

Figure 4. Expression levels of Col-1A1, Col-3A1, MMP-2/-9 and TGF- $\beta$ in CFs following rMIF treatment (20 and 40 nM) for 24 h. (A) Representative western blot analysis of ECM protein in treated cells; $\beta$-actin was used as the internal control. Densitometric analysis of protein expression of (B) Col-1A1, (C) Col-3A1, (D) MMP-9, (E) MMP-2 and (F) TGF- $\beta$ in treated cells and controls. ${ }^{* *} \mathrm{P}<0.01$ and ${ }^{*} \mathrm{P}<0.05$ vs. control. Each blot represents one of four experiments. rMIF, recombinant migration inhibitory factor; Col-1A1, collagen type 1, $\alpha 1$; Col-3A1, collagen type 3, $\alpha 1$; MMP-2/-9, matrix metalloproteinase-2/-9; TGF- $\beta$, transforming growth factor- $\beta$.

\section{Discussion}

The results of the present study demonstrated the following: i) Expression levels of collagen, MMP-2/-9 and TGF- $\beta$ were significantly increased in the left atrial tissues of patients with AF; ii) long-term treatment of rMIF promoted the proliferation of CFs; iii) PTK inhibitor inhibited the proliferation of CFs and reversed the increase in cell proliferation induced by rMIF; and iv) rMIF promoted the secretory function of CFs.

Atrial fibrosis is an important factor in initiating and maintaining AF, which is characterized by marked alterations in Col 1 and Col 3 synthesis/degradation, associated with disturbed MMP/tissue inhibitors of MMP systems and increased levels of TGF- $\beta$. Previous studies have revealed that the ECM changes are more severe in patients with permanent AF, compared with those in patients with SR $(3,15)$. In the present study, it was also found that, compared with the SR controls, the protein levels of Col-1A1/3A1, MMP-2/-9 and TGF- $\beta$ were significantly increased in the left atrial tissues of patients with AF.
It has been established in previous years that inflammation contributes to the atrial fibrosis and structural remodeling of AF. In the left atrium of patients with AF, the regions infiltrated by immune cells are positively correlated with left atrial fibrosis and left atrial dimension (10). In vitro, the direct cell-cell interaction between human monocytes and CFs can stimulate TGF- $\beta$-mediated myofibroblast activity and increase the local ECM remodeling (16). Certain inflammatory markers are indicators for the degree of left atrial fibrosis. As a novel marker of inflammation, high serum levels of YKL-40 are associated with the presence and more extensive left atrial fibrosis in patients with lone AF (12). In post-operative AF in rats with sterile pericarditis, anti-interleukin-17A monoclonal antibody has been shown to alleviate the inflammation and fibrosis, prolong refraction and markedly suppress the development of AF (17).

MIF, an important pro-inflammatory cytokine, is known to be involved in the inflammatory cardiovascular diseases. MIF can cause progress and instability of atherosclerotic plaques, increases the expression of intercellular adhesion molecule-1, and markedly induces the expression of MMP-9 $(18,19)$. 
MIF is also involved in the pathological process of myocarditis (20), and in cardiac dysfunction following burn injury and sepsis (21). It has also been demonstrated that the increased expression of MIF contributes to the development of electrical remodeling of atrial myocytes through downregulating the protein expression of L- and T-type calcium channels, and impairing the channel function $(8,13)$. However, the role of MIF in atrial fibrosis, and the proliferation and function of CFs remains to be fully elucidated. To investigate whether MIF is involved in the pathogenesis of atrial fibrosis, the present study detected the protein expression levels of MIF in left atrial homogenates from patients with AF or SR controls. It was demonstrated that the protein expression of MIF was significantly elevated in the atrial tissues from patients with $\mathrm{AF}$, which was positively correlated with the increased expression of ECM. These data implicate MIF in the upregulation of $\mathrm{ECM}$ in $\mathrm{AF}$.

In addition to excess ECM deposition, fibrosis is associated with fibroblast proliferation and differentiation into collagen-secreting myofibroblasts. To confirm whether MIF regulates the proliferation and function of CFs, and to address the possible mechanisms, the effects of rMIF on the proliferation and function of CFs were examined in the present study. These experiments indicated that treatment with human rMIF $(40 \mathrm{nM})$ for $48 \mathrm{~h}$ significantly promoted the proliferation of CFs.

Src, one of the proto-oncogenes encoding a membraneassociated, non-receptor PTK, has been implicated in the regulation of a wide range of cellular functions, including cell proliferation, survival and migration (22). The activation of Src can result in the promotion of survival and proliferation pathways, and even induce malignant tumors (v-src) (23). Src can be activated by several factors, including high glucose and inflammatory factors TNF- $\alpha$, IFN- $\gamma$ and MIF (8,24-26). In the present study, it was found that the specific Src antagonist, PP1, inhibited MIF-induced CF proliferation. In addition, $40 \mathrm{nM}$ rMIF was found to produce a marked increase in the levels of Src and the activation of Src kinase, compared with that in cells of the unstimulated group and $20 \mathrm{nM}$ rMIF treatment group. These results indicated that MIF promoted the proliferation of CFs through the Src kinase signaling pathway.

The present study also investigated the effect of MIF on the function of CFs, and found that rMIF treatment $(40 \mathrm{nM})$ for $24 \mathrm{~h}$ stimulated the production of fibrosis-related factors, including Col-1A1/3A1, MMP-2/-9 and TGF- $\beta$. Therefore, it was hypothesized that MIF, an important regulator of inflammation, stimulates the expression of Src kinase, which in turn promotes the proliferation of CFs and the production of factors associated with fibrosis, which contributes to the atrial fibrosis observed in AF.

There were a number of potential limitations in the present study. Firstly, atrial fibroblasts behave differently to ventricular fibroblasts in vitro and in vivo, with atrial fibroblasts showing more marked fibrotic and oxidative responses than ventricular fibroblasts $(27,28)$. In the present study, the cellular experiments were performed with mouse $\mathrm{CFs}$, which included atrial and ventricular fibroblasts, whereas the use of atrial fibroblasts is likely to be superior in investigations of AF. Secondly, with the increase of cell generation, the proportion of myofibroblasts increases, particularly for later passages $(>7)$. Therefore, cells with a similar proportion of myofibroblasts (passages 3-6) were preferable for use in the present study.

\section{Acknowledgements}

This study was supported by grants from the National Natural Science Foundation of China (grant nos. 81370295, 81470440 and 81670314), the Guangdong Natural Science Foundation (grant no. S2013010016256) and the Medical Science Foundation of Guangdong (grant no. A2013049).

\section{References}

1. Li D, Fareh S, Leung TK and Nattel S: Promotion of atrial fibrillation by heart failure in dogs: Atrial remodeling of a different sort. Circulation 100: 87-95, 1999.

2. Nattel S: How does fibrosis promote atrial fibrillation persistence: In silico findings, clinical observations and experimental data. Cardiovasc Res 110: 295-297, 2016.

3. Polyakova V, Miyagawa S, Szalay Z, Risteli J and Kostin S: Atrial extracellular matrix remodelling in patients with atrial fibrillation. J Cell Mol Med 12: 189-208, 2008.

4. Yue L, Xie J and Nattel S: Molecular determinants of cardiac fibroblast electrical function and therapeutic implications for atrial fibrillation. Cardiovasc Res 89: 744-753, 2011.

5. Miragoli M, Salvarani N and Rohr S: Myofibroblasts induce ectopic activity in cardiac tissue. Circ Res 101: 755-758, 2007.

6. Conway DS, Buggins P, Hughes E and Lip GY: Prognostic significance of raised plasma levels of interleukin- 6 and C-reactive protein in atrial fibrillation. Am Heart J 148: 462-466, 2004.

7. Guo Y, Lip GY and Apostolakis S: Inflammation in atrial fibrillation. J Am Coll Cardiol 60: 2263-2270, 2012.

8. Rao F, Deng CY, Wu SL, Xiao DZ, Yu XY, Kuang SJ, Lin QX and Shan ZX: Involvement of Src in L-type $\mathrm{Ca}^{2+}$ channel depression induced by macrophage migration inhibitory factor in atrial myocytes. J Mol Cell Cardiol 47: 586-594, 2009.

9. Deng H, Xue YM, Zhan XZ, Liao HT, Guo HM and Wu SL: Role of tumor necrosis factor-alpha in the pathogenesis of atrial fibrillation. Chin Med J (Engl) 124: 1976-1982, 2011.

10. Yamashita T, Sekiguchi A, Suzuki S, Ohtsuka T, Sagara K, Tanabe H, Kunihara T, Sawada H and Aizawa T: Enlargement of the left atrium is associated with increased infiltration of immune cells in patients with atrial fibrillation who had undergone surgery. J Arrhythm 31: 78-82, 2015.

11. Sonmez O, Ertem FU, Vatankulu MA, Erdogan E, Tasal A, Kucukbuzcu S and Goktekin O: Novel fibro-inflammation markers in assessing left atrial remodeling in non-valvular atrial fibrillation. Med Sci Monit 20: 463-470, 2014.

12. Canpolat U, Aytemir K, Hazirolan T, Özer N and Oto A: Serum YKL-40 as a marker of left atrial fibrosis assessed by delayed enhancement MRI in lone atrial fibrillation. Pacing Clin Electrophysiol 38: 1386-1395, 2015.

13. Rao F, Deng CY, Wu SL, Xiao DZ, Huang W, Deng H, Kuang SJ, Lin QX, Shan ZX, Liu XY, et al: Mechanism of macrophage migration inhibitory factor-induced decrease of T-type $\mathrm{Ca}(2+)$ channel current in atrium-derived cells. Exp Physiol 98: 172-182, 2013.

14. Wang WK, Wang B, Lu QH, Zhang W, Qin WD, Liu XJ, Liu XQ, An FS, Zhang Y and Zhang MX: Inhibition of high-mobility group box 1 improves myocardial fibrosis and dysfunction in diabetic cardiomyopathy. Int J Cardiol 172: 202-212, 2014.

15. Barth AS, Merk S, Arnoldi E, Zwermann L, Kloos P, Gebauer M, Steinmeyer K, Bleich M, Kääb S, Hinterseer M, et al: Reprogramming of the human atrial transcriptome in permanent atrial fibrillation: Expression of a ventricular-like genomic signature. Circ Res 96: 1022-1029, 2005.

16. Mewhort HE, Lipon BD, Svystonyuk DA, Teng G, Guzzardi DG, Silva C, Yong VW and Fedak PW: Monocytes increase human cardiac myofibroblast-mediated extracellular matrix remodeling through TGF- $\beta 1$. Am J Physiol Heart Circ Physiol 310: H716-H724, 2016.

17. Fu XX, Zhao N, Dong Q, Du LL, Chen XJ, Wu QF, Cheng X, Du YM and Liao YH: Interleukin-17A contributes to the development of post-operative atrial fibrillation by regulating inflammation and fibrosis in rats with sterile pericarditis. Int $\mathbf{J}$ Mol Med 36: 83-92, 2015. 
18. Kong YZ, Yu X, Tang JJ, Ouyang X, Huang XR, Fingerle-Rowson G, Bacher M, Scher LA, Bucala R and Lan HY: Macrophage migration inhibitory factor induces MMP-9 expression: Implications for destabilization of human atherosclerotic plaques. Atherosclerosis 178: 207-215, 2005.

19. Schmeisser A, Marquetant R, Illmer T, Graffy C, Garlichs CD Bockler D, Menschikowski D, Braun-Dullaeus R, Daniel WG and Strasser RH: The expression of macrophage migration inhibitory factor 1alpha (MIF 1alpha) in human atherosclerotic plaques is induced by different proatherogenic stimuli and associated with plaque instability. Atherosclerosis 178: 83-94, 2005.

20. Matsui Y, Okamoto H, Jia N, Akino M, Uede T, Kitabatake A and Nishihira J: Blockade of macrophage migration inhibitory factor ameliorates experimental autoimmune myocarditis. J Mol Cell Cardiol 37: 557-566, 2004.

21. Willis MS, Carlson DL, Dimaio JM, White MD, White DJ, Adams GA IV, Horton JW and Giroir BP: Macrophage migration inhibitory factor mediates late cardiac dysfunction after burn injury. Am J Physiol Heart Circ Physiol 288: H795-H804, 2005.

22. Cary LA, Klinghoffer RA, Sachsenmaier C and Cooper JA: SRC catalytic but not scaffolding function is needed for integrinregulated tyrosine phosphorylation, cell migration and cell spreading. Mol Cell Biol 22: 2427-2440, 2002.

23. Chen Q, Zhou Z, Shan L, Zeng H, Hua Y and Cai Z: The importance of Src signaling in sarcoma. Oncol Lett 10: 17-22, 2015.
24. Cai T, Kuang Y, Zhang C, Zhang Z, Chen L, Li B, Li Y, Wang Y, Yang H, Han Q and Zhu Y: Glucose-6-phosphate dehydrogenase and NADPH oxidase 4 control STAT3 activity in melanoma cells through a pathway involving reactive oxygen species, c-SRC and SHP2. Am J Cancer Res 5: 1610-1620, 2015.

25. Lin CC, Pan CS, Wang CY, Liu SW, Hsiao LD and Yang CM: Tumor necrosis factor-alpha induces VCAM-1-mediated inflammation via c-Src-dependent transactivation of EGF receptors in human cardiac fibroblasts. J Biomed Sci 22: 53, 2015.

26. Boekhoudt GH, McGrath AG, Swisher JF and Feldman GM: Immune complexes suppress IFN- $\gamma$-induced responses in monocytes by activating discrete members of the SRC kinase family. J Immunol 194: 983-989, 2015.

27. Burstein B, Libby E, Calderone A and Nattel S: Differential behaviors of atrial versus ventricular fibroblasts: A potential role for platelet-derived growth factor in atrial-ventricular remodeling differences. Circulation 117: 1630-1641, 2008.

28. Yeh YH, Kuo CT, Chang GJ, Qi XY, Nattel S and Chen WJ: Nicotinamide adenine dinucleotide phosphate oxidase 4 mediates the differential responsiveness of atrial versus ventricular fibroblasts to transforming growth factor- $\beta$. Circ Arrhythm Electrophysiol 6: 790-798, 2013. 